Case Report

Copyright (C) All rights are reserved by Arif Razzak

\title{
Panton-Valentine Leukocidin
}

\author{
Arif Razzak*, Andrew Jenkinson, Madhav Kittur and Steven Key \\ Oral and Maxillofacial Surgery SHO, Morriston Hospital, UK
}

*Corresponding author: Arif Razzak, Oral and Maxillofacial Surgery SHO, Morriston

Hospital, UK.

Received Date: September 20, 2019

Published Date: October 30, 2019

\begin{abstract}
We present a case of a Panton-Valentine Leukocidin (PVL) positive Staphylococcus aureus (SA) infection in an otherwise fit and well 21 year old man. This bacterial infection was preceded by a reactivation of a cold sore on his lower lip. The formation of an abscess and localised tissue necrosis prompted the search for this unusual and dangerous toxin.
\end{abstract}

\section{Introduction}

PVL is a toxin produced by Staphylococcus aureus. It induces soft tissue breakdown and necrosis increasing the virulence of the associated microorganism. It is commonly associated with cutaneous abscesses and carbuncles however it can cause complications such as bacteraemia, septic arthritis and necrotizing pneumonia. This case reports discusses a clinical scenario where a patient attended to a maxillofacial unit with an infection to his lip, which was found to be PVL positive.

\section{Clinical Presentation}

A 21 year old male university student presented to our maxillofacial department with a 10 day history of a soft tissue infection of his lower lip. Initially the infection arose from a recurrent cold sore which persisted for one week before the formation of an abscess, mucosal necrosis and associated cellulitis. Of note, this patent had previously experienced aggressive skin infections with carbuncle formation affecting other areas of his body.

A diagnosis of a secondary bacterial infection was made, the abscess was incised and drained under local anaesthetic and he was commenced on Intravenous antibiotics. The abscess recollected in the labial mucosa and extended into the buccal and vestibular spaces requiring further drainage under general anaesthetic. Human Immunodeficiency Virus and diabetes were excluded. However the lesion continued to persist (Figure 1).

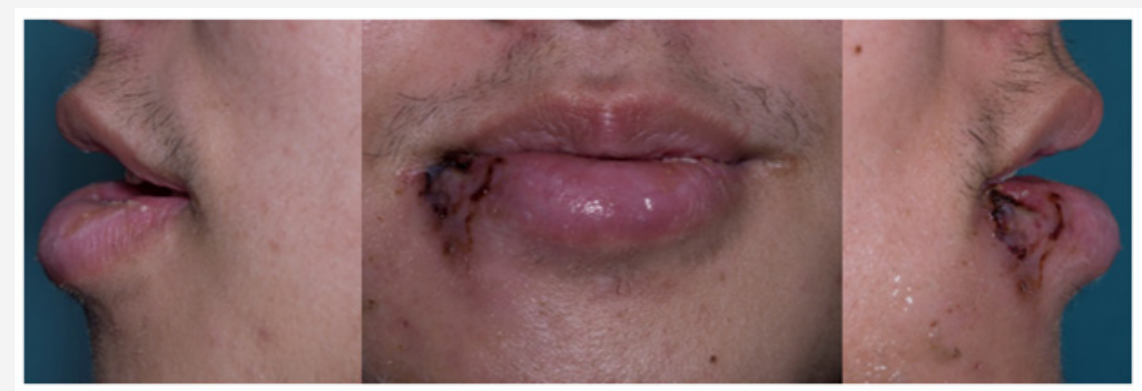

Figure 1: shows how the lesion appeared after the second drainage, showing areas of skin necrosis.

Methicillin Sensitive Staphylococcus aureus was grown from his lip and his blood cultures and given his clinical presentation there was a strong suspicion that this was a PVL toxin producing organism. A PVL assay was requested, the infection control team were contacted and the patient was barrier nursed. The patient was discharged home after 7 days of intravenous antibiotics and reviewed as an outpatient one week later. He had responded well to the prescribed treatment and his PVL assay demonstrated presence of the toxin. He was examined for systemic manifestations of the infection and consented for public health to perform contact tracing 
and an eradication regime to his relatives and recent contacts. The patient was followed up later and the lesion was found to be resolving (Figure 2).

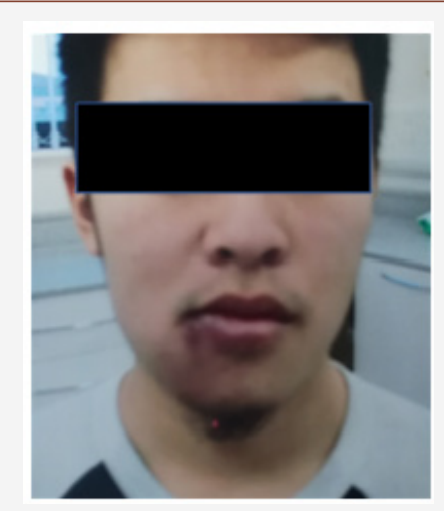

Figure 2: shows the appearance one week after discharge, note the resolving necrosis.

Panton-Valentine Leukocidin background. PVL is a poreforming cytotoxin produced by staphylococcus aureus which causes leukocyte lysis and tissue necrosis. These features produce a virulent organism capable of invasive, deep-seated infections. Typically, PVL producing staylococcal infections present with recurrent cutaneous lesions such as abscesses and carbuncles. They can also present with locally invasive features such as osteomyelitis and necrotising fasciitis. Systemic manifestations of the infection following haematogenous spread are septic arthritis and necrotising pneumonitis, and as such PVL infections are potentially fatal. Infections associated with PVL are uncommon. 720 cases were identified between 2005 and 2006 (7).

Risk factors for PVL infections include skin integrity, skin to skin contact, and sharing of items which may become contaminated, such as towels (7). For these reasons the infection is easily spread throughout sports teams and close relatives.

An important part of any PVL case is to ensure that the wider community is protected from the risk of infection. Decolonisation is required after the acute infection has subsided and an assessment of the patient's close relatives, sexual partners and other individuals who may be at risk should be made.

\section{Key learning points}

1. A High index of suspicion for PVL should arise when unusually invasive cutaneous infections present.

2. Exclude immunosuppression

3. Barrier nurse

4. Search for local and systemic manifestations of the infection

5. Involving public health teams when PVL is identified to perform contact tracing and eradication

\section{Acknowledgement}

None.

\section{Conflict of Interest}

No conflict of interest.

\section{References}

1. Deurenberg RH, Stobberingh EE (2008) The evolution of Staphylococcus aureus. Infection, genetics \& evolution 8(6): 747-763.

2. Muttaiyah S, Coombs G, Pandey S, Reed P, Ritchie S, et al. (2010) Incidence, risk factors, and outcomes of Panton-Valentine leukocidinpositive methicillin-susceptible Staphlococcus aureus infections in Auckland, New Zealand. J Clin Microbiol 48(10): 3470-3474.

3. Lina G, Piémont Y, Godail-Gamot F, Bes M, Peter M, et al. (1999) Involvement of Panton-Valentine leukocidin-producing Staphylococcus aureus in primary skin infections and pneumonia. Clin Infect Dis 29(5): 1128-1132.

4. Damasco PV, Chamon RC, Barbosa ATL, Da Cunha S, Aquino JHW, et al. (2012) Involvement of methicillin-susceptible Staphylococcus aureus related to sequence type 25 and harbouring PVL genes in a case of carotid cavernous fistula after community-associated sepsis. J Clin Microbiol 50(1): 196-198.

5. Kaneko J, KamioY (2004) Bacterial two-component and heteroheptameric pore-forming cytolytic toxin: structure, pore-forming mechanism, and organization of the genes. Biosci Biotechnol Biochem 68(5): 981- 1003.

6. Varshney A, Martinez LR, Hamilton SM, et al. (2010) Augmented production of Panton-Valentine leukocidin toxin in methicillin-resistant and methicillinsusceptible Staphylococcus aureus is associated with worse outcome in a murine skin infection model. J Infect Dis 201(1): 9296.

7. Health Protection agency (2008) Guidance on the diagnosis and management of PVL-associated Staphylococcus aureus infections. $2^{\text {nd }}$ edn, (PVL-SA) in England. PVL subgroup of the steering group on healthcare associated infection. 